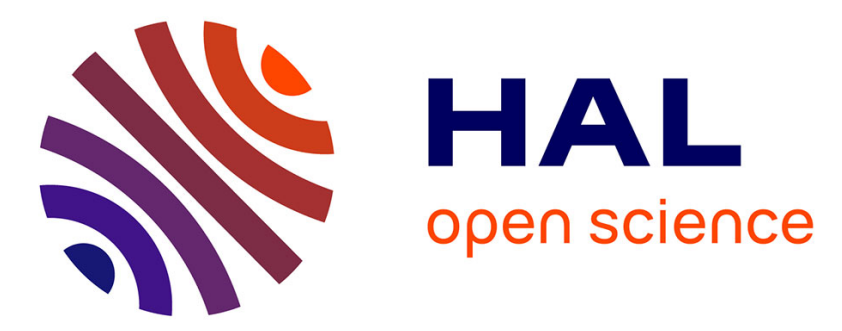

\title{
The role of dispersion forces on the atomic structure of glassy chalcogenides: The case of GeSe4 and GeS4
}

Ziyad Chaker, Guido Ori, Christine Tugène, Sébastien Le Roux, Mauro Boero, Carlo Massobrio, Évelyne Martin, Assil Bouzid

\section{- To cite this version:}

Ziyad Chaker, Guido Ori, Christine Tugène, Sébastien Le Roux, Mauro Boero, et al.. The role of dispersion forces on the atomic structure of glassy chalcogenides: The case of GeSe4 and GeS4. Journal of Non-Crystalline Solids, 2018, 499, pp.167-172. 10.1016/j.jnoncrysol.2018.07.012 . hal-02312769

\section{HAL Id: hal-02312769 \\ https://hal.science/hal-02312769}

Submitted on 17 Dec 2021

HAL is a multi-disciplinary open access archive for the deposit and dissemination of scientific research documents, whether they are published or not. The documents may come from teaching and research institutions in France or abroad, or from public or private research centers.
L'archive ouverte pluridisciplinaire HAL, est destinée au dépôt et à la diffusion de documents scientifiques de niveau recherche, publiés ou non, émanant des établissements d'enseignement et de recherche français ou étrangers, des laboratoires publics ou privés. 


\title{
The role of dispersion forces on the atomic structure of glassy chalcogenides: the case of $\mathrm{GeSe}_{4}$ and $\mathrm{GeS}_{4}$
}

\author{
Ziyad Chaker, Guido Ori, Christine Tugène, Sébastien Le Roux, Mauro Boero, Carlo Massobrio \\ Institut de Physique et Chimie des Matériaux de Strasbourg, Université de Strasbourg, \\ CNRS UMR 7504, F-67034 STRASBOURG Cedex 2, France \\ Evelyne Martin \\ Université de Lille, CNRS, Centrale Lille, ISEN, Université Valenciennes, UMR 8520-IEMN, F-59000 Lille, France
}

Assil Bouzid

Chaire de Simulation à l'Échelle Atomique (CSEA), École Polytechnique Fédérale de Lausanne (EPFL), CH-1015 Lausanne, Switzerland

\begin{abstract}
Recent first-principles molecular dynamics simulations (FPMD) results on two chalcogenide glasses (glassy $\mathrm{GeS}_{4}$ and $\mathrm{GeSe}_{4}$ ) are revisited by accounting explicitly for van der Waals (vdW) dispersion forces. This effort is motivated by the observation that such contributions were found to be important in the case of glassy $\mathrm{GeTe}_{4}$ while they were negligible for another disordered chalcogenide system, liquid $\mathrm{GeSe}_{2}$. With the present results, we provide additional evidence intended to establish under which conditions and for which systems the consideration of dispersion forces plays a role in determining the atomic structure. For these purposes we employ two different dispersion schemes used in conjunction with the BLYP (Becke, Lee, Yang and Parr) exchange-correlation functional. The first is the DFT-D2 vdW correction introduced by Grimme et al. ([1]) while the second is the $\mathrm{vdW}_{W}$ approach based on the Wannier functions analysis [2]. The atomic structures obtained agree well with the ones obtained by Bouzid et al. [3] without considering dispersion forces. Due to the vdW interactions, glassy $\mathrm{GeSe}_{4}$ features a higher number of Ge fourfold coordinated. The two vdW approaches also agree to a large extent, exceptions occurring for some moderate differences in the intensity of the peaks in the Ge-Ge pair correlation function.
\end{abstract}

Key words: Car-Parrinello Molecular Dynamics (CPMD), Atomic structure, di-chalcogenides, van der Waals 


\section{Introduction}

The inclusion of dispersion (van der Waals, vdW) forces in first-principles molecular dynamics (FPMD) treatments of disordered systems is of paramount importance to assess and improve the predictive power of these approaches for various kinds of bonding characteristics. In the case of water, for instance, vdW 5 interactions are crucial to obtain realistic properties for such universal solvent [4]. Turning to chalcogenides network-forming disordered materials, it appears that dispersion forces are more likely to play a role in $\mathrm{Ge}_{x} \mathrm{Te}_{1-x}$ than in $\mathrm{Ge}_{x} \mathrm{Se}_{1-x}$ ones, as highlighted by a recent set of FPMD results [5]. This is due to the impact of predominant bonding components (of ionic or iono-covalent origin) screening more effectively dispersion effects in $\mathrm{Ge}_{x} \mathrm{Se}_{1-x}$ than in $\mathrm{Ge}_{x} \mathrm{Te}_{1-x}$ systems.

In spite of these achievements, the issue of dispersion forces in chalcogenides disordered networks cannot be considered as completely settled and deserves further investigations. This is due to the paucity of the cases examined so far and the availability of several vdW recipes, not necessarily equally performing and of general applicability $[6,7]$.

These concepts have been exemplified by our recent investigation devoted to liquid $\mathrm{GeSe}_{2}$ [8]. In that paper, the authors employed two distinct descriptions for the dispersion forces, namely the Grimme-D2 or the maximally localized Wannier function scheme (detailed later in the section devoted to the methodology). This second scheme is more profoundly rooted into density functional theory since the electronic structure, obtained within the selected DFT-GGA framework, is used to compute the maximally localized Wannier functions (MLWF) on which the van der Waals scheme is based. In what follows the results corresponding to each one of these two schemes will be referred to as $\mathrm{vdW}_{G}$ (Grimme) and $\mathrm{vdW}_{W}$ (Wannier) respectively. We remind that in the case of liquid $\mathrm{GeSe}_{2}$, by calculating properties such as partial structure factors, pair correlation functions, bond angle distribution, and number of corner vs edge sharing connections, it was found that the maximally localized Wannier function scheme is more reliable than the Grimme-D2 scheme in reproducing existing first-principles results. In particular, the Grimme-D2 scheme worsens the agreement with experiments in the case of the Ge-Ge pair correlation function. The main message of that study was that the impact of dispersion forces on disordered chalcogenides is not necessarily the same when adopting different theoretical schemes.

Based on the above findings, this paper is intended to address the two following issues: (a) whether there are other disordered chalcogenides liquid or glasses (among those already investigated) for which dispersion forces cannot be neglected and, related to this, (b) to establish the sensitivity to the specific vdW recipe employed, in analogy with the case of liquid $\mathrm{GeSe}_{2}$ [8]. To attain these purposes we focus here on glassy $g$ - $\mathrm{GeSe}_{4}$ and $g$ - $\mathrm{GeS}_{4}$ by performing a comparative study and by considering three set of results, namely those obtained by Bouzid et al. [3] with no vdW interactions account (NovdW) and the present new data for which we employed the $\mathrm{vdW}_{G}$ and $\mathrm{vdW}_{W}$ schemes. In short, our results show that dispersion forces 
are at the origin of minor changes in the structural properties of both glasses, $g$-GeSe ${ }_{4}$ being the one most affected in terms of Ge tetrahedral coodination.

This paper is organized as follows. In section II, we provide the methods and computational details of the calculations performed in this work with a special emphasis on the two schemes of vdW. In section

The second scheme $\left(\mathrm{vdW}_{W}\right)$ considered makes use of the Wannier functions analysis [14] to compute the maximally localized Wannier functions [15] (MLWFs) and use these for the $a b$ initio evaluation of the vdW vdW corrections are detailed. For each system and each vdW scheme, we compare the total pair correlation function and the total structure factors $\mathrm{S}(k)$ to the experimental data available. Then we move to the partial Faber-Ziman structure factors and to the partial pair correlation functions. Finally, we draw a detailed table of the different coordination numbers obtained for the atomic structures we considered. In section IV, general conclusions are proposed on the role of vdW corrections for this pair of glassy chalcogenide systems.

\section{Computational methods}

Our simulations were performed within the Car-Parrinello [9] molecular dynamics (CPMD) scheme as implemented in the CPMD code [10]. We employ the exchange density-functional proposed by Becke [11] and the one due to Lee, Yang and Parr for the correlation part [12] (BLYP). This Generalized Gradient Approximation (GGA) is known to provide an accurate description of the network topology in the case of binary glassy chalcogenide systems [6]. For the treatment of the core-valence interactions, we adopted a norm-conserving pseudo-potential according to the description of Troullier and Martins [13]. A plane wave basis set is chosen for the representation of the valence electrons with a corresponding energy cutoff of 30 Ry $[6,3,8]$. The Brillouin zone integration is restricted to the $\Gamma$ point.

As mentioned in the introduction, we consider two types of implementations of the van der Waals (vdW) corrections. The first is the DFT-D2 formula proposed by Grimme [1] which is a DFT-based formulation in which parameters are self-consistently tuned in conjunction with the specific exchange-correlation functional considered. We recall that, although empirical, the parameters involved in this formulation tuned on different functionals, including the one used in this work, and benchmarked on a wealth of different systems from simple molecules to complex reactive surfaces and chalcogenides. No experimental parameters are considered in the construction of this specific vdW correction. Its inclusion does not affect any stage of the Kohn-Sham equations, thus preserving the first-principle character of all electronic structure calculations. However, the Grimme formalism does not allow for any update of the vdW interactions resulting from the changes in the electronic structure that occur during the dynamical evolution. maximally localized Wannier functions [15] (MLWFs) and use these for the ab initio evaluation of the vdW 
energy. The inclusion of $\mathrm{vdW}_{W}$ correction using MLWFs, first introduced in Ref. [2], is written as [4]:

$$
E_{v d W}=-\sum_{n<l} f\left(r_{n l}\right) \frac{C_{6, n l}}{r_{n l}^{6}}
$$

In this case, the full system is partitioned into fragments on the basis of its connectivity in a given configuration so that $\mathrm{r}_{n l}$ is the distance between two MLWF centers on pairs of fragments while $\mathrm{f}\left(\mathrm{r}_{n l}\right)$ is a damping function. The MLWFs allow to perform unbiased partitioning of the charge density while their computation can be performed on the fly during the CPMD run with a reasonably low computational cost [4]. The MLWF centers are defined as:

$$
x_{n}=-\frac{L}{2 \pi} \Im m\left\{\log \left(\left\langle w_{n}|\exp (-2 i \pi \cdot x / L)| w_{n}\right\rangle\right)\right\}
$$

for the $\mathrm{x}$-direction but similar expressions can be written for the other two spatial directions. The spreads of the MLWF are used to evaluate the $\mathrm{C}_{6, n l}$ coefficients according to the procedure proposed by Silvestrelli et al. [16]. Although the analytical form of the vdW energy (1) is similar to the one of the DFT-D2 scheme, the $\mathrm{C}_{6}$ coefficients are calculated directly from the first principles electronic structure calculation at each molecular dynamics step.

During our first-principles molecular dynamics runs, the temperature of the ions was controlled via a Nosé-Hoover thermostat $[17,18]$ and a similar method has been applied to control the evolution of the temperature of the fictitious electronic degrees of freedom along the scheme proposed by Blöchl and Parrinello [19]. An integration step of 7 au $(0.168 \mathrm{fs})$ has been used for the $\mathrm{GeS}_{4}$-vdW $\mathrm{vW}_{G}$ case while a slightly lower time-step of $5 \mathrm{au}(0.120 \mathrm{fs})$ is used for the three other cases $\left(\mathrm{GeSe}_{4}-\mathrm{vdW} G, \mathrm{GeS}_{4}\right.$-vdW $\left.\mathrm{vd}_{W}, \mathrm{GeSe}_{4}-\mathrm{vdW}_{W}\right)$, ensuring an optimal control of the conserved quantities all along the molecular dynamics trajectories.

Our $\mathrm{GeX}_{4}(\mathrm{X}=\mathrm{Se}$ or $\mathrm{S})$ glassy systems are periodic structures with a cubic primitive cell containing 480 atoms (96 Ge and $384 \mathrm{X}$ ) and a side equal to $24.82 \AA$ for $\mathrm{GeS}_{4}$ and $24.87 \AA$ for $\mathrm{GeSe}_{4}$. By taking as initial configuration one extracted from the trajectory produced in the NovdW case, extended trajectories were implemented to lose memory of this starting condition [6]. Both systems were constructed and studied within the framework of the CPMD-vdW $\mathrm{v}_{G}$ or CPMD-vdW ${ }_{W}$ methods applying a specific thermal cycle. For the $\mathrm{vdW}_{G}$ case, the initial systems were brought at a ionic temperature of $\mathrm{T}=900 \mathrm{~K}$ and equilibrated during about $40 \mathrm{ps}$, the temperature was then lowered to $\mathrm{T}=700 \mathrm{~K}$ for $55 \mathrm{ps}$ and finally lowered to room temperature $(\mathrm{T}=300 \mathrm{~K})$ to produce the trajectory $(10 \mathrm{ps})$ exploited for the structural analysis. A shorter cycle have been considered for the $\mathrm{vdW}_{W}$ calculations including: 1 ps at $300 \mathrm{~K}, 1.5$ ps at $600 \mathrm{~K}, 10 \mathrm{ps}$ at $900 \mathrm{~K}, 5$ ps at $600 \mathrm{~K}$ and finally a trajectory larger than $10 \mathrm{ps}$ at $300 \mathrm{~K}$ for both $\mathrm{GeX}_{4}$ models to produce the data used in this work. 
To characterize the atomic structures of the glassy materials studied within our FPMD calculations, the partial pair correlation functions are defined as the ratio between the density $\rho_{\alpha \beta}(\mathrm{r})$ in a shell of thickness $d r$ and the total density of the system $\rho_{0}$

$$
g_{\alpha \beta}(r)=\frac{\rho_{\alpha \beta}(r)}{\rho_{0}}
$$

90 of each $\mathrm{g}_{\alpha \beta}(\mathrm{r})$ can be taken as a measure of the bond length $\mathrm{r}_{\alpha \beta}$. By integrating up to the first minimum of the corresponding pair correlation function, one obtains the partial coordination number $\mathrm{N}_{\alpha \beta}$.

The total pair correlation function $\mathrm{g}_{T}(\mathrm{r})$ is obtained as follows

$$
g_{T}(r)-1=\sum_{\alpha=1}^{2} \sum_{\beta=1}^{2} \frac{c_{\alpha} c_{\beta} b_{\alpha} b_{\beta}}{\langle b\rangle^{2}}\left[g_{\alpha \beta}(r)-1\right]
$$

The quantities $c_{\alpha}$ and $b_{\alpha}$ are, respectively, the atomic fractions and the coherent neutron scattering lengths of Ge $(8.185 \mathrm{fm})$, Se $(7.97 \mathrm{fm})$ and $\mathrm{S}(2.847 \mathrm{fm})$. The mean coherent neutron scattering length $\langle b\rangle$ is defined as

$$
\langle b\rangle=c_{G e} b_{G e}+c_{X} b_{X}
$$

with $\mathrm{X}=$ Se or S. The reciprocal space counterpart of the partial pair correlation functions are the FaberZiman (FZ) partial structure factors obtained by applying a Fourier transform to $\mathrm{g}_{\alpha \beta}$

$$
S_{\alpha \beta}^{F Z}(k)-1=\frac{4 \pi \rho_{0}}{k} \int_{0}^{\infty} r\left[g_{\alpha \beta}(r)-1\right] \sin (k r) d r
$$

The total neutron structure factor $\mathrm{S}_{T}(\mathrm{k})$ is a weighted sum of these partial quantities

$$
S_{T}(k)-1=\sum_{\alpha=1}^{2} \sum_{\beta=1}^{2} \frac{c_{\alpha} c_{\beta} b_{\alpha} b_{\beta}}{\langle b\rangle^{2}}\left[S_{\alpha \beta}^{F Z}(k)-1\right]
$$

\section{Results}

\section{A - Neutron total structure factor and pair distribution function}

95 . In figure 1, we report the total structure factors for both $g$ - $\mathrm{GeSe}_{4}$ and $g$-GeS 4 . We include the results obtained with no consideration of vdW interactions [3] as well as the experimental data obtained by Petri [20], Salmon [21] and bychkov [22].

We observe that all the methods used succeed in reproducing a signature (more or less intense depending on the system) of the first sharp diffraction peak (FSDP) at about $\mathrm{k} \simeq 1 \AA^{-1}$. This means that the vdW forces do not play a major role in the establishment of an intermediate range. The different level of intensity recorded for the FSDP in the total neutron structure factor of $g$ - $\mathrm{GeS}_{4}$ and $g$ - $\mathrm{GeSe}_{4}$ can be correlated to the sizes of the rings existing in these glasses, as shown by Bouzid et al. [3]. Indeed, through the $n$-atoms rings 


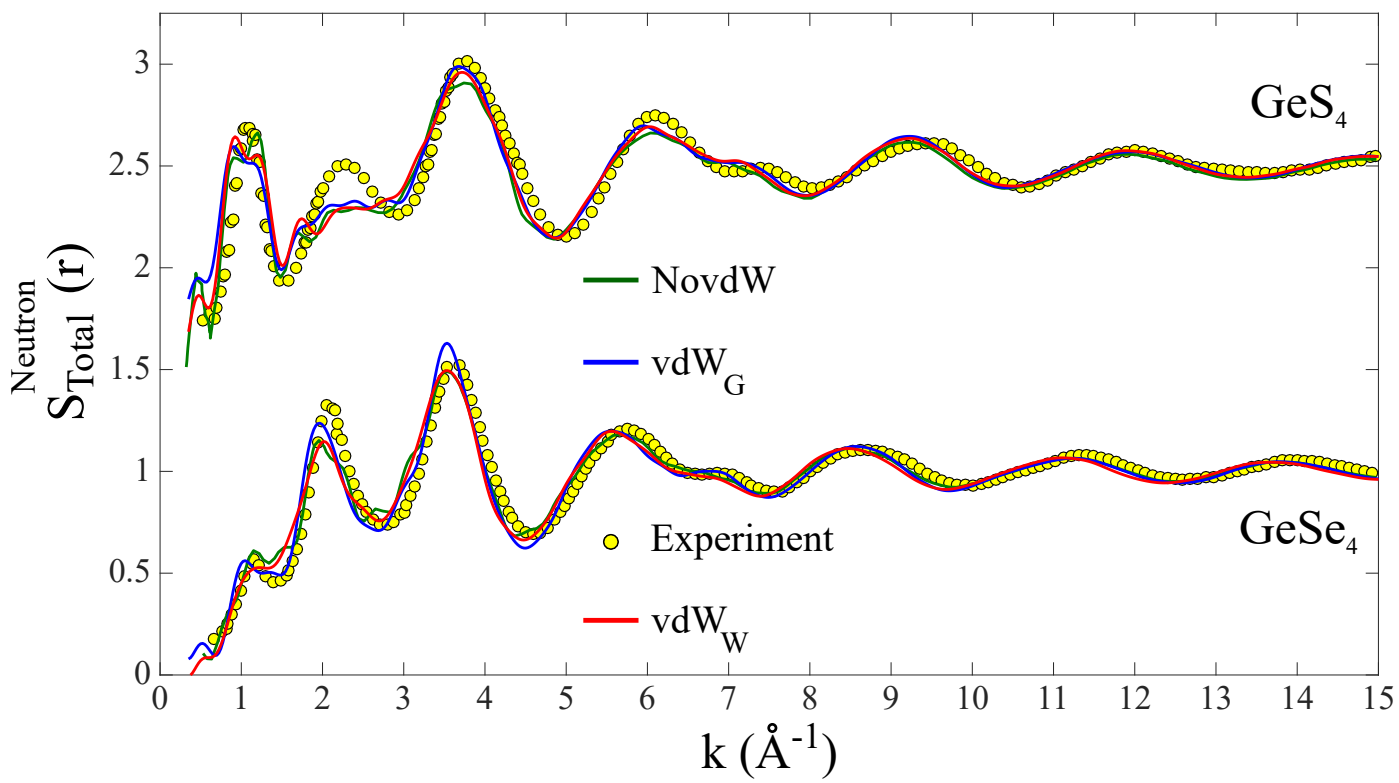

Figure 1: Total neutron structure factors for both $\mathrm{GeS}_{4}$ (top panel shifted upwards by 1.5) and $\mathrm{GeSe}_{4}$ (bottom panel) evaluated as a Fourier transform of the total pair correlation function. The "NovdW" results correspond to those with no inclusion of vdW interactions (green curves) as reported by Bouzid et al. [3]. The experimental data (circle symbols) are those reported by Petri [20], Bychkov [22] and Salmon[21]. Two different forms of the van der Waals corrections are given: vdW $\mathrm{W}_{G}$ using the Grimme approach [1] (blues solid lines) and $\mathrm{vdW}_{W}$ using the Wannier approach introduced by Silvestrelli et al. [2] (red solid lines)

analysis, it was demonstrated that the occurrence of six-membered rings is much more frequent in $g$-GeS 4 than in $g$-GeSe 4 . In addition, the presence of higher ring sizes in $g$ - $\mathrm{GeSe}_{4}(\mathrm{n}=10,13,15)$ can be associated to ring diameters beyond the first two nearest neighbors (> $5 \AA$ ). Thus, such Ge-S motifs are responsible for the differences between the FSDP of $g$ - $\mathrm{GeS}_{4}$ and $g$ - $\mathrm{GeSe}_{4}$ (figure 1). Globally, the total neutron structure factors are equally well reproduced by all schemes, with the exception of the second main peak in the $\mathrm{GeS}_{4}$ system. However, for this feature, the underestimate persists regardless of the presence or absence of dispersion forces.

Similar considerations can be expressed when focusing on the total pair correlation function (see figure 2). The experiments are due to Petri [20], Bychkov [22] and Salmon [21]. We refer to the work of Bouzid et al. [3] for the FPMD calculations performed with no inclusion of vdW interactions. The experimental data sets referred to were obtained by applying a Fourier transformation to the total neutron structure factors using, in both cases, the upper limits of integration of the experimental finite measurement window function of the diffractometer: $\mathrm{k}_{\max }\left(g-\mathrm{GeSe}_{4}\right)=19.95 \AA^{-1}, \mathrm{k}_{\max }\left(g-\mathrm{GeS}_{4}\right)=49.95 \AA^{-1}$. As shown in Ref. [3], this procedure of calculation can lead to spurious oscillations obtained in both cases in the region $r<2 \AA$ (figure 2). Our calculations do not show such artifacts since our calculations were performed directly in real space 
by applying the definition of the pair correlation function. As a first observation, we note that the first sharp maximum corresponding to the nearest neighbor distance is very well reproduced both by $\mathrm{vdW}_{G}$ (blue lines)

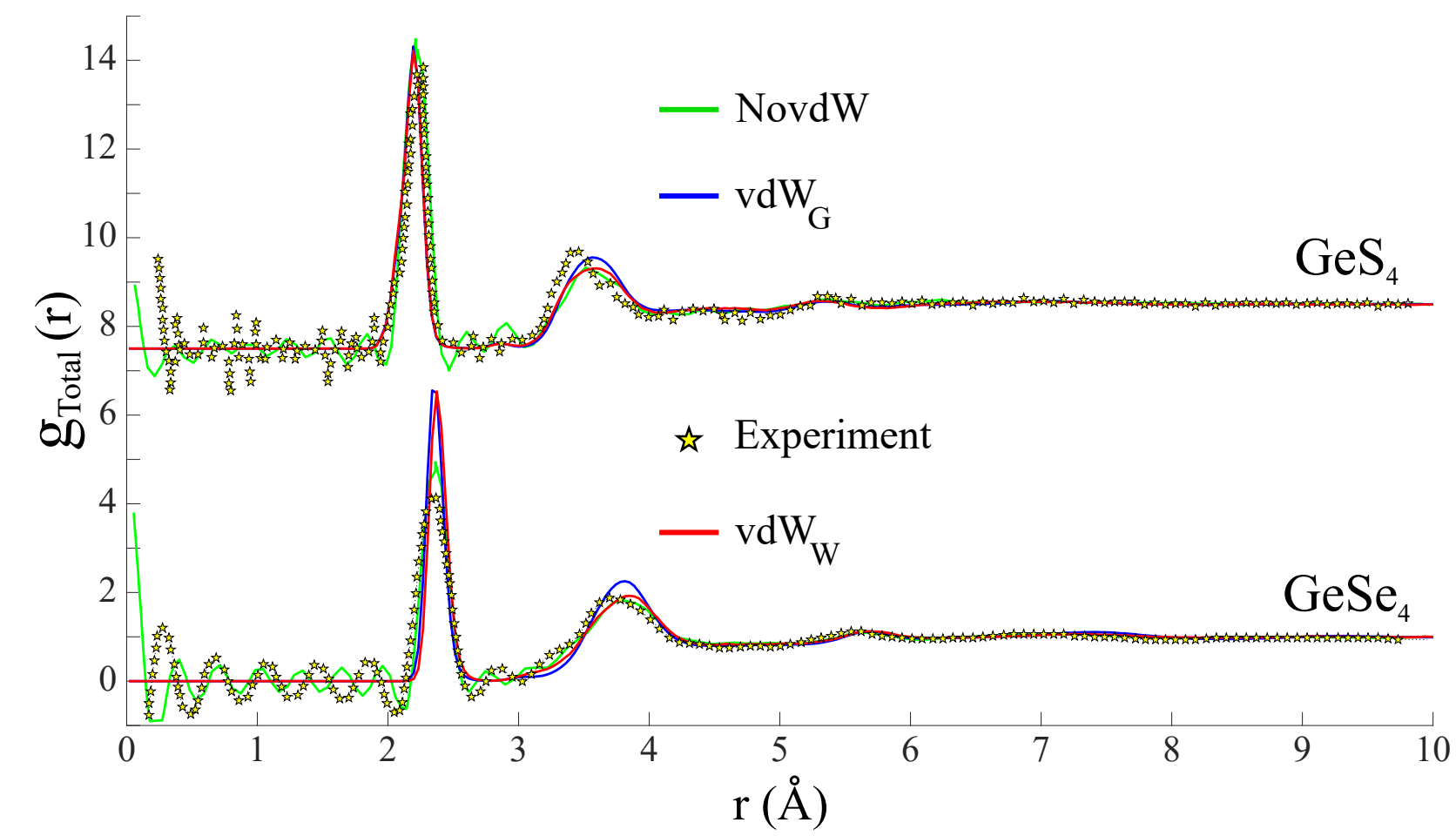

Figure 2: Total pair correlation function for $\mathrm{GeS}_{4}$ (top panel shifted upwards by $7.5 \AA$ ) and $\mathrm{GeSe}_{4}$ (bottom panel) calculated with no inclusion of vdW interactions (green curves) as reported by Bouzid et al. [3] and compared to our results including the two different forms of the van der Waals corrections discussed in the text: vdW $\mathrm{vd}_{G}$ (blues solid lines) and the vdW $\mathrm{vW}_{W}$ (red solid lines). The experimental data (yellow symbols) correspond to those reported by Petri [20], Bychkov [22] and Salmon[21]. 


\section{B Partial structure factors}

. In order to go further in the analysis of the atomic structure, we analyze the partial Faber-Ziman (FZ) structure factors for each atomic pair. The partial structure factors for both systems are reported in figure 3. Focusing on the FSDP visible for $g-\mathrm{GeSe}_{4}$ in $\mathrm{S}_{G e G e}^{F Z}$, we observe that the same effect is reproduced by both $\operatorname{vdW}_{G}$ and $\operatorname{vdW}_{W}$ calculations and by the NovdW approach used in ref. [3]. The height of the FSDP intensities is reduced in the $g$-GeS $\mathrm{G}_{4}$ case with a split pattern appearing around $\mathrm{k} \simeq 1 \AA^{-1}$, mostly to be ascribed to statistical noise. We observe that the FSDP is obtained in the Ge-S and Ge-Se partial structure factors but not in the Se-Se and S-S case. Therefore, one can draw similar conclusions as the ones obtained in the NovdW case, namely that the peak obtained in the total structure factor at $\mathrm{k} \simeq 1 \AA^{-1}$ (figure 1 ) is due to the Ge-Ge and Ge-X $(\mathrm{X}=\mathrm{Se}, \mathrm{S})$ correlations that are at origin of intermediate range order.

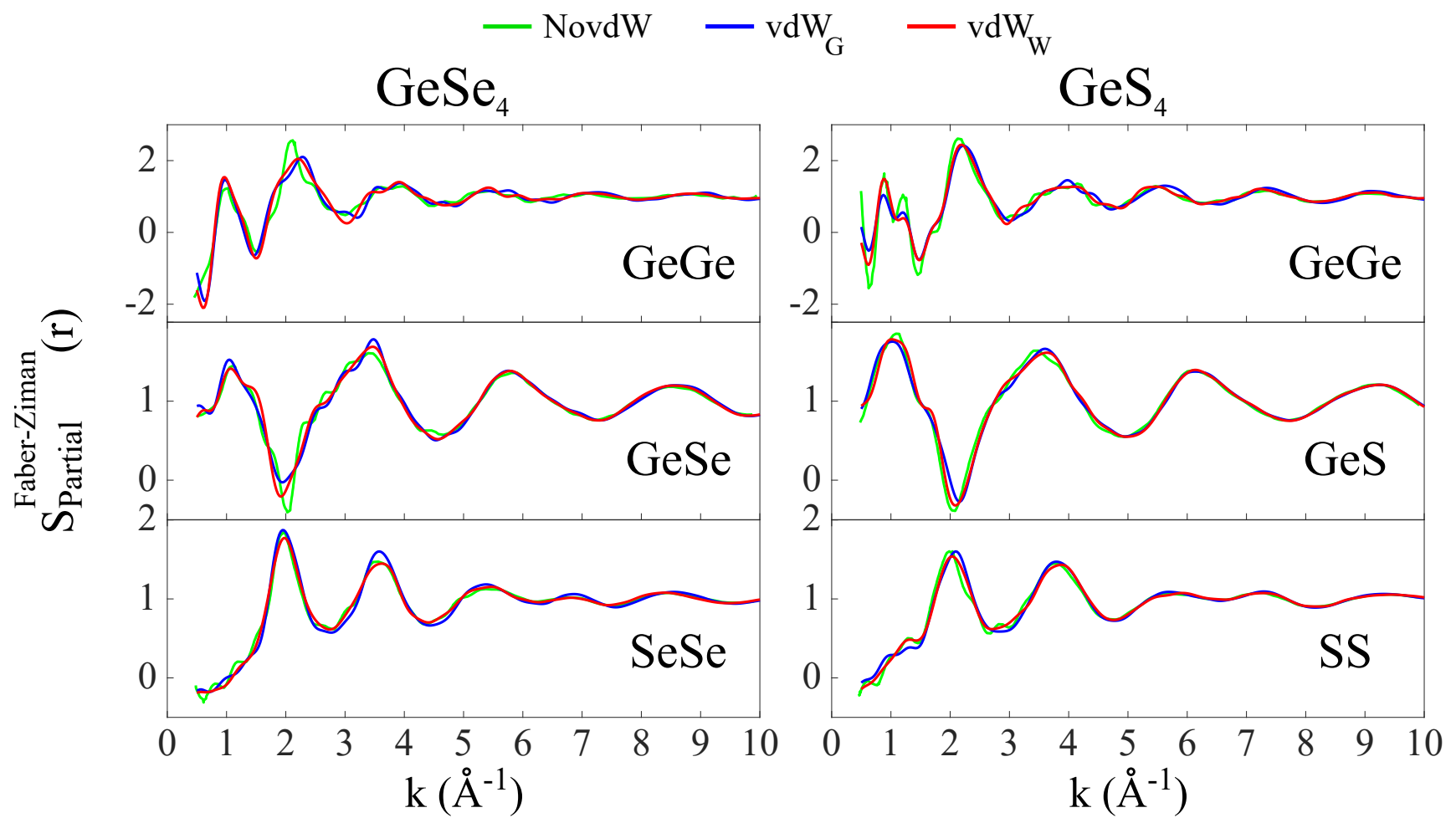

Figure 3: The Faber-Ziman partial structure factors for both glassy binary chalcogenides: $\mathrm{GeSe}_{4}$ (left panel) and GeS 4 (right panel), calculated with no inclusion of vdW interactions (green curves) as reported by Bouzid et al. [3]. The calculations including the two different forms of the van der Waals corrections are vdW $\mathrm{w}_{G}$ (blues solid lines) and the vdW $\mathrm{W}_{W}$ (red solid lines)

\section{Partial pair correlation functions}

. We describe the real space properties of $g$ - $\mathrm{GeSe}_{4}$ and $g$ - $\mathrm{GeS}_{4}$ by calculating the partial pair correlation functions of the different atomic pairs. These results are reported in figure 4.

For the Ge-X pair correlation functions, the results obtained by the three methods are practically super- 


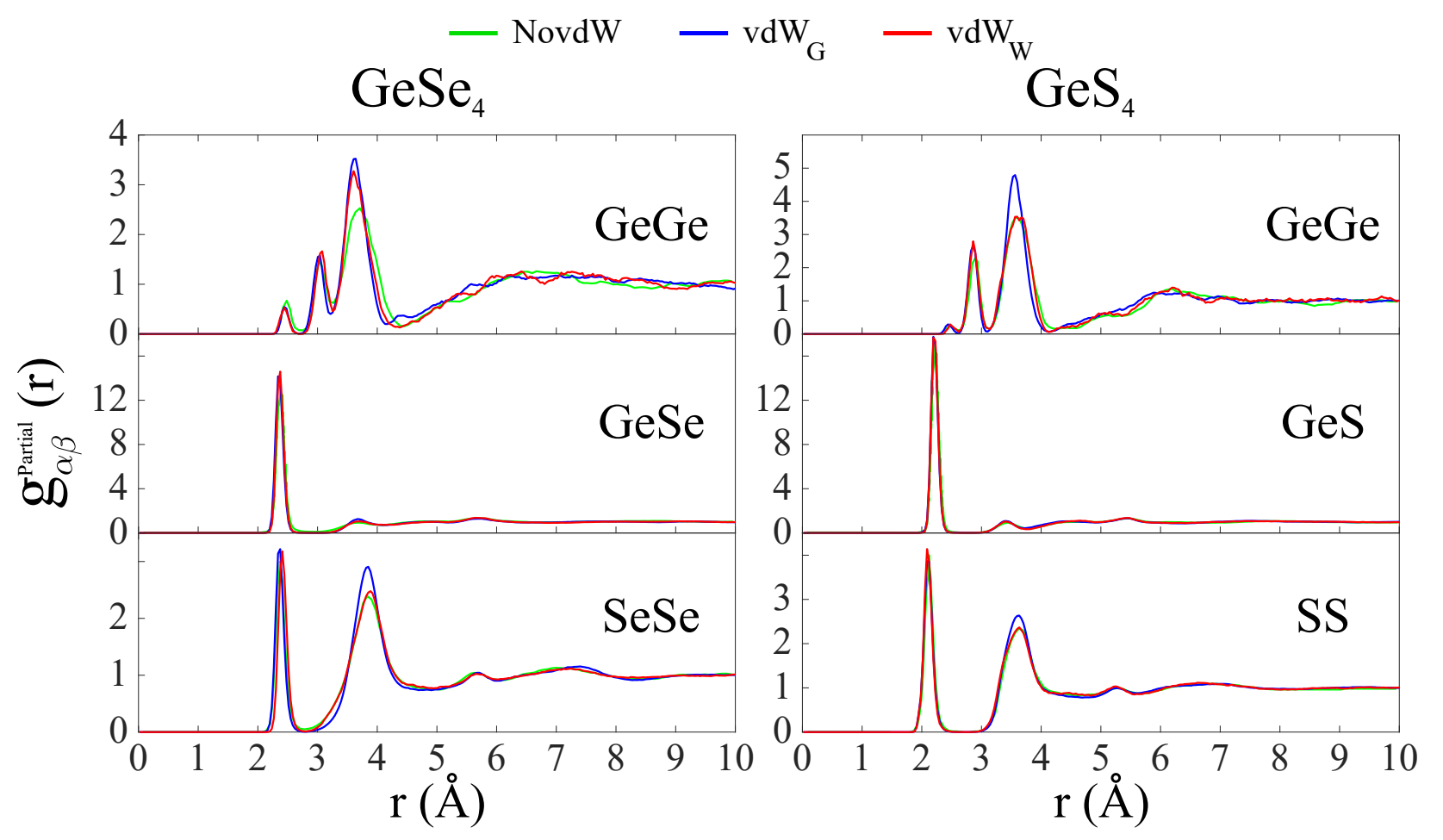

Figure 4: The partial pair correlation functions for the atomic pairs of $g$-GeSe 4 (left panel) and $g$-GeS 4 (right panel). Results with no inclusion of vdW interactions (green curves) as reported by Bouzid et al. [3] are compared to those including van der Waals, $\operatorname{vdW}_{G}$ (blues solid lines) and the $\mathrm{vdW}_{W}$ (red solid lines)

imposed for both systems reproducing the same differences between $\mathrm{GeS}_{4}$ and $\mathrm{GeSe}_{4}$ materials found in the report by Bouzid et al. [3].

In the Ge-Ge case, the first small peak observed at $\mathrm{r} \simeq 2.45 \AA$ is obtained with the three calculation methods at the same position for both systems. In terms of its intensity, the three approaches reproduce the same intensity for $g-\mathrm{GeSe}_{4}\left(\mathrm{~g}_{G e G e}^{G e S e} \simeq 0.5\right)$ and for $g-\mathrm{GeS}_{4}\left(\mathrm{~g}_{G e G e}^{G e S_{4}} \simeq 0.2\right)$. This peak is the signature of the presence of homopolar Ge-Ge bonds. Two other peaks are noticeable. The first corresponds to the edge-sharing (ES) tetrahedra and the second to corner-sharing (CS) tetrahedra. These peaks are obtained (figure 4) at values of $\mathrm{r} \simeq 3.0 \AA$ and $\mathrm{r} \simeq 3.6 \AA$ for $\mathrm{GeSe}_{4}$, slightly differing from the GeS 4 case in which they are obtained at $\mathrm{r} \simeq 2.9 \AA$ and $\mathrm{r} \simeq 3.6 \AA$. A close look at the CS-tetrahedra population of $\mathrm{g}_{G e G e \text { (peaks }}$ at $\mathrm{r} \simeq 3.6 \AA$ ) reveals that the $\mathrm{vdW}_{W}$ approach tend to match the $\operatorname{vdW}_{G}$ results in $g$-GeSe 4 with a higher intensity of this peak in comparison to the NovdW calculation (green line). However, the opposite situation is obtained for $g$ - $\mathrm{GeS}_{4}$ system, for which the $\mathrm{vdW}_{G}$ scheme overestimates the intensity of Ge-Ge correlations at $\mathrm{r} \simeq 3.6 \AA$. On the contrary vdW $W$ gives an intensity comparable to the case with no vdW inclusion.

The situation for $g_{X X}$ is again indicative of small differences between the vdW $W_{G}$ and vdW $W_{W}$ results. The 

$0.04)$

In this Table (top part), we also report the coordination numbers in terms of the contributions (percentage $\mathrm{n}_{\alpha}(l)$ ) of each specific environment. In the case of $g$ - $\mathrm{GeSe}_{4}$, the deviation from the tetrahedral

first peak indicative of nearest neighbor Se-Se distances $(\mathrm{r} \simeq 2.4 \AA)$ and the one corresponding to S-S bonds $(\mathrm{r} \simeq 2.1 \AA)$ are very close in the three models. However, for the second peak located at $\mathrm{r} \simeq 3.8 \AA\left(\mathrm{GeSe}_{4}\right)$ and $\mathrm{r} \simeq 3.6 \AA\left(\mathrm{GeS}_{4}\right)$, the $\mathrm{vdW}_{W}$ calculation tend to reproduce the NovdW results rather than the $\mathrm{vdW}_{G}$ ones. Such a trend is similar to what already observed in the case of liquid $\mathrm{GeSe}_{2}$ [8]. It appears that Ge-Ge correlations are the most sensitive to the presence and/or the quality of the dispersion forces considered. While the three models are broadly consistent and the changes observed of moderate extent, the impact on Ge-Ge correlations of the dispersion forces will be an issue worth pursuing with additional calculations.

\section{D - Local order: coordination numbers}

. Despite the trace of some moderate level of sensitivity to the presence of dispersion forces, it appears that the properties examined so far do not provide any evidence on the occurrence of any striking effect in $g$ $\mathrm{GeSe}_{4}$ and $g$ - $\mathrm{GeS}_{4}$, either when adopting the dispersion forces or when selecting the Wannier scheme instead of the DFT-D2 one. We complete the structural analysis presented above by the investigation of the local coordination obtained through the different calculation schemes considered. These results are reported in the Table. In the bottom part of this table, we provide the detailed coordination numbers $\mathrm{N}_{\alpha \beta}$ for both $g$-GeSe $\mathrm{G}_{4}$ (left part) and $g$-GeS 4 systems as well as the resulting total ones $\mathrm{N}_{\alpha}(\alpha=\mathrm{Ge}$, Se or $\mathrm{S})$. These coordination numbers provide information on the number of neighbors for each atom and to the kind of structural unit formed with the given neighbors, by identifying the chemical nature of each interatomic linkage.

Ge atoms are found to be four fold coordinated $\left(\mathrm{N}_{G e} \simeq 4\right)$ mainly due to the tetrahedral arrangement of Se and $\mathrm{S}$ around Ge species in $\mathrm{GeSe}_{4}$ and $\mathrm{GeS}_{4}$. This is confirmed by all $\mathrm{N}_{G e X}$ values found to be very close to $4 . \mathrm{X}(\mathrm{X}=\mathrm{Se}, \mathrm{S})$ atoms are two-fold coordinated for both materials with heteropolar (X-Ge) and homopolar $\mathrm{X}-\mathrm{X}$ bonds having coordination numbers are very close to $1\left(\mathrm{~N}_{X G e} \simeq \mathrm{N}_{X X} \simeq 1\right)$.

The three calculations methods give almost identical results indicating that the contribution of vdW interactions inclusion does not affect the characteristic tetrahedral network of these materials. Nevertheless, the NovdW scheme leads to a higher amount of homopolar Ge-Ge bonds in the $g$-GeSe $\mathrm{G}_{4}$ system. Indeed, $\mathrm{N}_{G e G e}^{G e S e_{4}}$ values (0.36 for NovdW) differ from the values very close to 0 found in the present work $\left(\mathrm{N}_{G e G e}=\right.$

arrangement is quite limited when dispersion forces are included, values of the percentage of Ge atoms fourfold coordinated to Se atoms approaching closely the unity. No other effects due to the different account of dispersion forces are found, the different amounts of the corresponding coordination units being very close. 


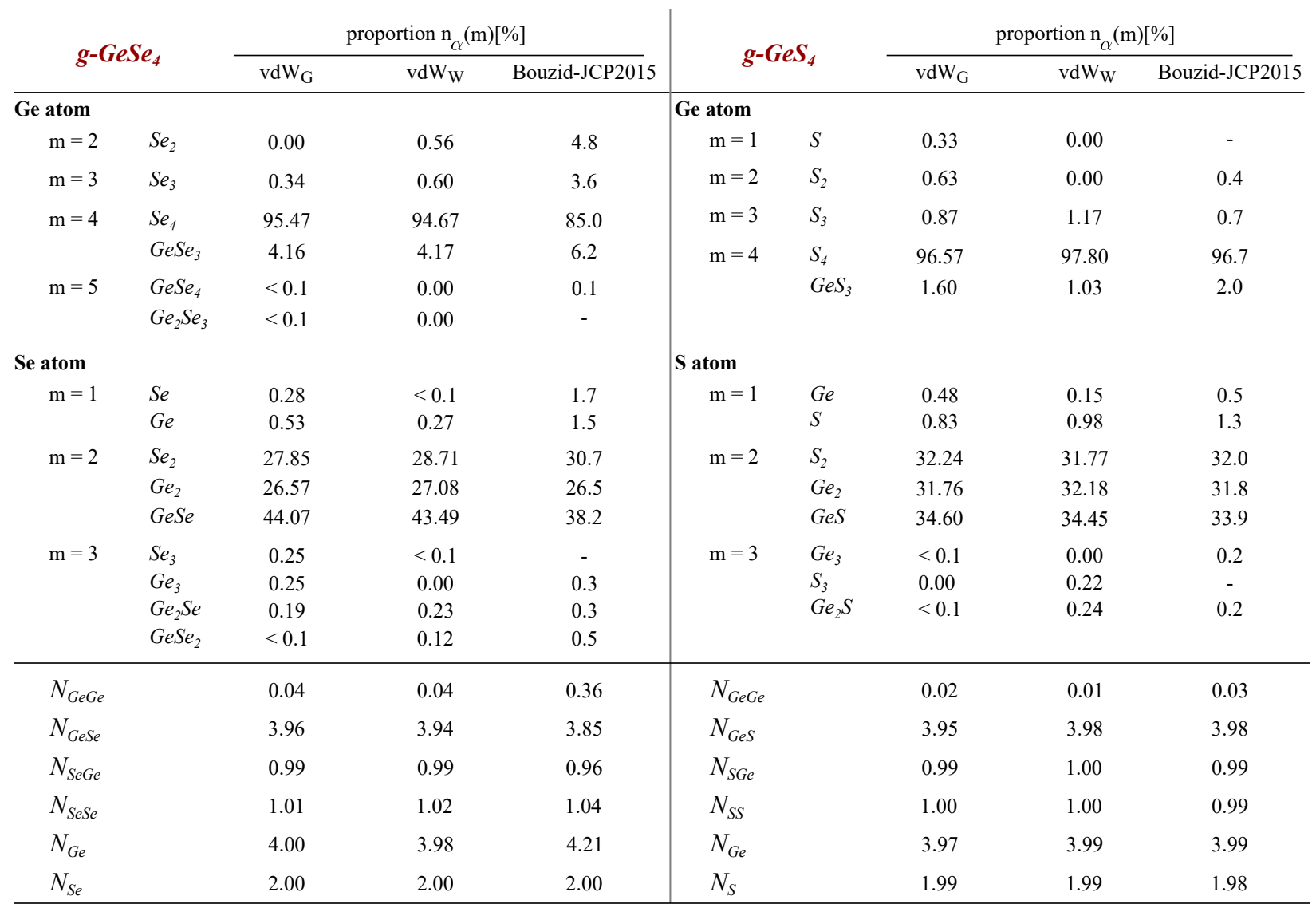

Table: Percentage $\mathrm{n}_{\alpha}(\mathrm{l})$ of the different coordination units (l) in glassy $\mathrm{GeSe}_{4}$ (left part) and $\mathrm{GeS}_{4}$ (right part) for the specie $\alpha$ (Ge, S or Se). These quantities have been calculated including neighbors separated by a cutoff corresponding to the first minimum in the partial pair correlation functions. The corresponding average atomic pairs and total coordination numbers are given in the bottom part of the table.

In the case of $\mathrm{GeS}_{4}$, there are even less differences among the three set of results, the percentage number of Ge atoms fourfold coordinated to Se atoms being already very close to the unity in the NovdW case. It looks like at this level of description, involving the first shell of coordination of these disordered systems, the dispersion forces can be safely neglected for $g$ - $\mathrm{GeS}_{4}$ and they bring limited and yet appreciable changes for $g$ - $\mathrm{GeSe}_{4}$, by reducing the number of Ge atoms that deviate from the tetrahedral environment.

\section{Conclusion}

We revisited the structural properties of glassy $\mathrm{GeSe}_{4}$ and $\mathrm{GeS}_{4}$ systems by relying on first-principles molecular dynamics. We undertake such a study since other disordered chalcogenide systems were found sensitive to the inclusion of dispersion forces and, to a lesser extent, also sensitive to the kind of theoretical recipe employed to model such contributions [6]. In this work we presented a detailed comparison between 
two specific approaches designed for the proper inclusion of vdW forces contributions, namely, the vdW $\mathrm{due}$

to Grimme [1] and relying on a semi-empirical description and a fully first-principles based method (vdW $\left.{ }_{W}\right)$ taking advantage of the the Wannier functions formalism [15]. Based on the results obtained for the total and partial pair correlation functions, total and partial structure factors, supplemented by an analysis of the neighbor environments (coordination units with the neighbors of each species) we could not detect any sizable effect of the dispersion forces on the main features of the networks. However, there is one effect that is worth pointing out, this being the tendency of both $\mathrm{vdW}$ schemes to enhance the fourfold coordination environment in glassy $\mathrm{GeSe}_{4}$, by producing an atomic structure that turns out very similar to that of glassy $\mathrm{GeS}_{4}$. In conclusion, the body of our results brings new information on the structure of these glasses when compared to what is already available in the literature. The new outcome is twofold: on the one hand, lack of substantial sensitivity to dispersion forces, once again to be contrasted to the case of some Te-based chalcogenides [5]. On the other hand, the inclusion of dispersion forces attenuates the differences between the network topologies of glassy $\mathrm{GeSe}_{4}$ and $\mathrm{GeS}_{4}$, both of them made by a highly predominant presence of Ge atoms fourfold coordinated with very few Ge-Ge homopolar bonds.

\section{References}

\section{References}

[1] S. Grimme, Semiempirical GGA-type density functional constructed with a long-range dispersion correction, Journal of computational chemistry 27 (15) (2006) 1787-1799.

[2] P. L. Silvestrelli, Van der Waals interactions in DFT made easy by Wannier functions, Physical Review Letters 100 (5) (2008) 053002.

[3] A. Bouzid, S. Le Roux, G. Ori, M. Boero, C. Massobrio, Origin of structural analogies and differences between the atomic structures of $\mathrm{GeSe}_{4}$ and $\mathrm{GeS}_{4}$ glasses: A first principles study, The Journal of Chemical Physics 143 (3) (2015) 034504.

[4] T. Ikeda, M. Boero, Role of van der Waals corrections in first principles simulations of alkali metal ions in aqueous solutions, The Journal of Chemical Physics 143 (19) (2015) 194510.

[5] M. Micoulaut, A. Piarristeguy, H. Flores-Ruiz, A. Pradel, Towards accurate models for amorphous GeTe: Crucial effect of dispersive van der Waals corrections on the structural properties involved in the phase-change mechanism, Physical Review B 96 (18) (2017) 184204.

[6] A. Bouzid, C. Massobrio, M. Boero, G. Ori, K. Sykina, E. Furet, Role of the van der Waals interactions and impact of the exchange-correlation functional in determining the structure of glassy $\mathrm{GeTe}_{4}$, Physical Review B 92 (13) (2015) 134208. 
[7] Z. Chaker, G. Ori, M. Boero, C. Massobrio, E. Furet, A. Bouzid, First-principles study of the atomic structure of glassy $\mathrm{Ga}_{10} \mathrm{Ge}_{15} \mathrm{Te}_{75}$, Journal of Non-Crystalline Solids.

[8] E. Lampin, A. Bouzid, G. Ori, M. Boero, C. Massobrio, Impact of dispersion forces on the atomic structure of a prototypical network-forming disordered system: The case of liquid $\mathrm{GeSe}_{2}$, The Journal of Chemical Physics 147 (4) (2017) 044504.

[9] R. Car, M. Parrinello, Unified approach for molecular dynamics and density-functional theory, Physical Review Letters 55 (22) (1985) 2471.

[10] J. Hutter, M. Parrinello, D. Marx, P. Focher, M. Tuckerman, W. Andreoni, A. Curioni, E. Fois, U. Rothlisberger, P. Giannozzi, et al., Computer code CPMD, IBM Zurich Research Laboratory and MPI für Festkörperforschung 2001.

[11] A. D. Becke, Density-functional exchange-energy approximation with correct asymptotic behavior, Physical Review A 38 (6) (1988) 3098.

[12] C. Lee, W. Yang, R. G. Parr, Development of the Colle-Salvetti correlation-energy formula into a functional of the electron density, Physical Review B 37 (2) (1988) 785.

[13] N. Troullier, J. L. Martins, Efficient pseudopotentials for plane-wave calculations. II. operators for fast iterative diagonalization, Physical Review B 43 (11) (1991) 8861.

[14] G. H. Wannier, The structure of electronic excitation levels in insulating crystals, Physical Review 52 (3) (1937) 191.

[15] P. L. Silvestrelli, N. Marzari, D. Vanderbilt, M. Parrinello, Maximally-localized Wannier functions for disordered systems: Application to amorphous silicon, Solid State Communications 107 (1) (1998) 7-11.

[16] A. Ambrosetti, P. Silvestrelli, van der waals interactions in density functional theory using wannier functions: Improved $\mathrm{C}_{6}$ and $\mathrm{C}_{3}$ coefficients by a different approach, Physical Review B 85 (7) (2012) 073101.

[17] S. Nosé, A unified formulation of the constant temperature molecular dynamics methods, The Journal of Chemical Physics 81 (1) (1984) 511-519.

[18] W. G. Hoover, Canonical dynamics: equilibrium phase-space distributions, Physical Review A 31 (3) (1985) 1695.

[19] P. E. Blöchl, M. Parrinello, Adiabaticity in first-principles molecular dynamics, Physical Review B 45 (16) (1992) 9413. 
[20] I. Petri, P. Salmon, The topology of $\mathrm{Ge}_{x}-\mathrm{Se}_{1-x}(0<\mathrm{x}<0.4)$ glasses, Physics and Chemistry of Glasses 43 (2002) 185-190.

[21] P. S. Salmon, Structure of liquids and glasses in the Ge-Se binary system, Journal of Non-Crystalline Solids 353 (32) (2007) 2959-2974.

[22] E. Bychkov, M. Miloshova, D. Price, C. Benmore, A. Lorriaux, Short, intermediate and mesoscopic range order in sulfur-rich binary glasses, Journal of Non-Crystalline Solids 352 (1) (2006) 63-70. 